$5 A$

\title{
The Intervening Galaxies Hypothesis of the Absorption Spectra of Quasi-Stellar Objects: Some Statistical Studies
}

By

Debiprosad Duari and Jayant V. Narlikar
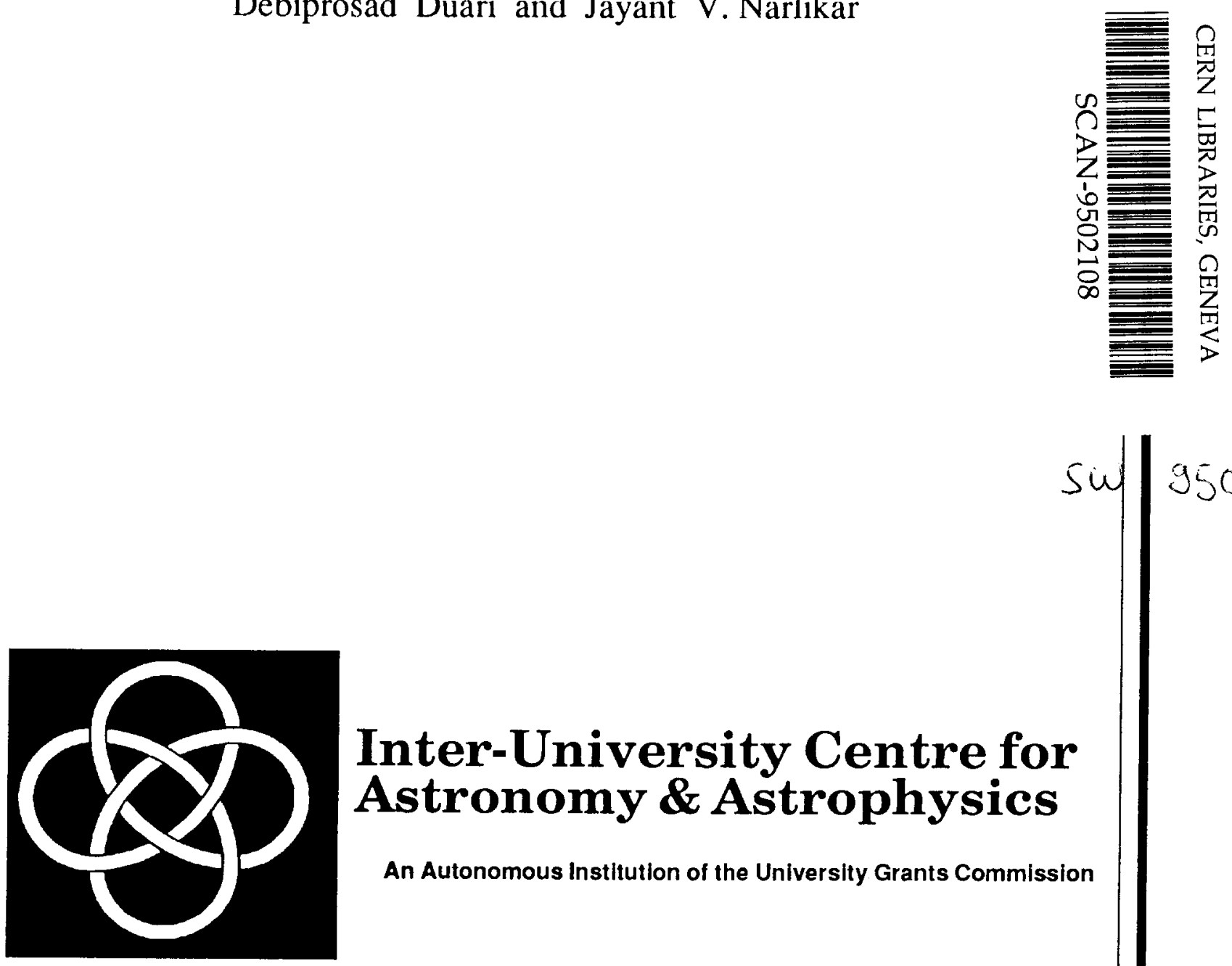

Inter-University Centre for Astronomy \& Astrophysics

An Autonomous Institution of the University Grants Commission 


\section{THE INTERVENING GALAXIES HYPOTHESIS}

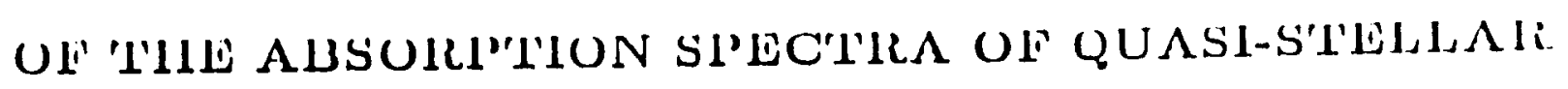

\section{OBJECTS : SOME STATISTICAL STUDIES}

by

Debiprosad Duari \& Jayant V. Narikar

Inter-University Centre for Astronomy and Astrophysics

Post Bag 4, Ganeshkhind, Pune 411 00i, India. 


\begin{abstract}
This paper examines, in the light of the available data, the hypothesis that the heavy element absorption line systems in the spectra of QSOs originate through en-route absorption by intervening galaxies, halos etc. Several statistical tests are applied in two different ways to compare the predictions of the intervening galaxies hypothesis(IGH) with actual observations. The database is taken from a recent 1991 compilation of absorption line systems by Junkkarinen, Hewitt and Burbidge. Although, prima facie, a considerable gap is found between the predictions of the intervening galaxies hypothesis and the actual observations despite inclusion of any effects of clustering and some likely selection effects, the gap narrows after invoking evolution in the number density of absorbers and allowing for the incompleteness and inhomogeneity of samples examined. On the latter count the gap might be bridgeable by streching the parameters of the theory. It is concluded that although the intervening galaxies hypothesis is a possible natural explanation to account for the absorption line systems and may in fact do so in several cases, it seems too simplistic to be able to account for all the available data. It is further stressed that the statistical techniques described here will be useful for future studies of complete and homogenous samples with a view to deciding the extent of applicability of the IGH.
\end{abstract}

Subject headings : quasars-absorption spectra; intervening galaxies hypothesis 


\section{Introduction}

The absorption spectra of quasistellar objects contain valuable clues for cosmology. As far back as 1965, Bahcall and Salpeter ${ }^{1}$ had discussed the various ways in which intervening absorbing medium can affect the spectra of distant sources. One of these was the appearance of absorption systems due to intervening galaxies. By now the current wisdom recognizes three kinds of absorption systems, each possibly having a different origin :

1. The Broad Absorption Line (BAL) systems which appear as broad troughs in the continuum blueward of the corresponding emission lines. Lines of high state of ionization are found, e.g. C IV, Si IV, N V, O VI, etc. apart from Lyman alpha. It is generally accepted that these systems arise in the quasar itself from absorption by material ejected by the quasar [Sargent $\left.{ }^{2}\right]$. The troughs have widths of upto $0.1 c$ and they extend upto equivalent velocity $\leqslant 0.2 c$ relative to the emission redshift.

2. The Lyman alpha systems appearing as sharp lines often densely populated, provided the quasar has large enough emission redshift. Such systems appear to the blue side of the Ly $\alpha$ emission line. It is commonly assumed that these lines arise from primordial intergalactic clouds distributed between us and the quasar.

3. The sharp lines due to $H$ and heavier elements which can arise in a tenuous gas of near solar compositon. The absorption redshifts can differ considerably from the emission redshifts with $z_{e m}-z_{a b}$, ranging from Doppler speeds of 
infall at a few thousand $\mathrm{kms}^{-1}$ to those of outflow as large as $\sim 0.8 c$. The majority view is that these lines are caused by absorption in or around galaxies that lie between us and the quasar. Thus $z_{a b}$ corresponds to the redshift of the intervening galaxy.

In this paper we will be concerned with this third class of absorption line systems, and shall refer to the above hypothesis of their origin as the intervening galaxies hypothesis (IGH in brief). It has been discussed by various authors from the early days of the discovery of absorption line systems ( $c f$, for example, Wagoner ${ }^{3}$, Bahcall and Spitzer ${ }^{4}$, Burbidge ${ }^{5}$, Roeder ${ }^{6}$ ). Sargent et $\mathrm{al}^{7,8,9}$ have found good agreement of the observed data with the IGH. Moreover, Bergeron \& Boissé ${ }^{10}$, and Steidel \& Dickenson ${ }^{11}$ have found optical fuzzes having redshifts similar to the redshifts of the absorption systems in and around the han if int to the quasars.

Despite persuasive cases of this kind, the IGH still remains to be universally established. Since it has the merit of making a testable prediction, viz. that the absorption redshift equals that of the intervening absorber, a useful insight into its validity is provided by statistical studies of large samples of such systems. These studies seek to relate the distributions of absorption systems to the distributions of absorbing material at the corresponding redshifts. This approach was first used by Burbidge et $\mathrm{al}^{5}$ and it involved relating the multiplicity of absorption systems in QSOs to the distribution of the absorbing galaxies, halos, clouds etc. Burbidge et $\mathrm{al}^{3}$ found multiple redshift systems far more frequent than the prediction of the IGH, and so concluded in favour of the intrinsic absorption hypothesis as a possible explanation of the absorption sytems. Recently, Borgeest and Mehlert ${ }^{12}$ 
have carried out statistical analysis of highly displaced C IV sytems, taken from the line lists of five surveys found in the literature and concluded that their result makes the association hypothesis for at least a large fraction of the absorbers more probable.

In this paper we will first redo the test of Burbidge et $\mathrm{al}^{5}$, as well as a few other statistical tests with the considerably augmented data on the quasars available today. We will then perform another test on the two more frequently found absorption systems, C IV and $\mathrm{Mg}$ II wherein, using the IGH we calculate the expected distribution of the absorption redshifts of either system and then compare it with the observed distribution. Could the differences between the observed and expected distributions be explained by chance fluctuations?

The database considered here is the Junkkarinen, Hewitt and Burbidge ${ }^{13}$ catalogue of absorption systems referred to as the JHB'91. the catalogue contains 353 quasars with 1106 absorption lines. If we argue that those lines which have $\left|z_{e m}-z_{a b s}\right| \leq 0.01\left(1+z_{e m}\right)$ might be intrinsic to the quasars, then eliminating these leaves 315 quasars with 953 absorption systems. Thus multiple absorption systems are common, the average per quasar exceeding 3 . The sample used by us is the largest available and carefully documented collection of absorption systems of QSOs. It is, however, neither complete nor homogenous.

The wisdom of using incomplete and/or inhomogeneous databases may be questioned. As Bahcall ${ }^{14}$ had emphasized in the early days when such comparisons were being attempted that in general different observers use different levels of sensitivity in detecting absorption lines at different redshifts and that even the 
same observer in the same quasar uses different sensitivities in detecting lines in different wavelength ranges. Thus no two quasars can apparently be treated by the same statistical yardstick. Extreme care therefore needs to be taken to sort out the selection effects that might affect these comparisons.

Our purpose in doing the analysis reported here is two-fold. First, we show that with due attention paid to incompleteness and inhogeneities and with adequate allowances for selection effects, something significant still emerges from our analysis. Secondly, we hope that regardless of the data the approaches used in the tests and the statistical techniques employed here have a lasting value and will come in handy as and when future observations deliver large, complete and more homogenous samples. Thus the conclusions drawn here are indicative rather than decisive, while the technignes an analysis should be seen as useful tools for examining future data.

\section{The Distribution of Multiple Systems}

\subsection{Theory}

For completeness we briefly go over the analysis of Burbidge et al ${ }^{5}$. Let $z_{1}, z_{2}, \ldots, z_{N}$ be the redshifts of the quasars in a given sample. For a typical $i^{\text {th }}$ member of the sample, say, with redshift $z_{i}$, the chance of interception by an intervening galaxy would depend on the following parameters :

(i) the number $n(z)$ of absorbers at the redshift $z<z_{i}$,

(ii) the projected cross section $\sigma$ of the absorber perpendicular to the line of sight, and

(iii) the cosmological parameters $H_{0}$ and $q_{0}$. 
Rather than repeat the details of the calculations, we quote the result derived by Wagoner ${ }^{3}$ and Bahcall \& Peebles $^{15}$ and used by Burbidge et $\mathrm{al}^{5}$ in the case of no evolution, that the number of intercepting galaxies in the redshift range $(z, z+d z)$ is given by :

$$
d \mu(z)=\frac{D_{0}}{l_{0}} \frac{(1+z) d z}{\left(1+2 q_{0} z\right)^{\frac{1}{2}}}=\frac{c\langle\sigma n\rangle}{H_{0}} \frac{(1+z) d z}{\left(1+2 q_{0} z\right)^{1 / 2}}
$$

where $D_{0}=c / H_{0}$, and $l_{0}$ is given by $l_{0}=\langle\sigma n\rangle_{z=0}^{-1}$. In this section we will use $H_{0}=50 \mathrm{~km} \mathrm{~s}^{-1} \mathrm{Mpc}^{-1}$.

Next, Burbidge et al estimated $l_{0}$ as follows. The Schechter luminosity function $^{16}$ for galaxies is given by

$$
d n=\phi^{*}\left(\frac{L}{L^{*}}\right)^{-5 / 4} \exp \left(-\frac{L}{L^{*}}\right) d\left(\frac{L}{L^{*}}\right)
$$

where $L^{*}$ is the fiducial luminosity corresponding to $M_{B}^{*}=-20.6, \phi^{*}=5 \times 10^{-3}$ $\mathrm{Mpc}^{-3}$. For galactic discs the Holmberg ${ }^{17}$ radius-luminosity relation gives

$$
\sigma=\sigma^{*}\left(\frac{L}{L^{*}}\right)^{5 / 6}=\pi R^{* 2}\left(\frac{L}{L^{*}}\right)^{3 / 6}
$$

where a typical value for $R^{*}$ may be taken as $20 \mathrm{kpc}$ (for M 101 optical radius, with $M_{B}^{*}=-21$ ). Allowing for the fact that only spirals and irregulars may be the right candidates for producing heavy element absorption lines and making estimates for projection perpendicular to line of sight, as well as for the fraction of the disc occupied by absorption clouds, Burbidge et al (op. cit.)estimated 


$$
l_{0}=\left[\sigma^{*} \phi^{*} \Gamma(7 / 12)\right]^{-1} \approx 240 \mathrm{Gpc}
$$

where $\Gamma(x)$ is the gamma function.

Estimates of $l_{0}$ are less well determined for larger absorbers. For example, for galactic coronae $R^{*} \approx 40 \mathrm{kpc}$ while for galactic halos $R^{*} \approx 100 \mathrm{kpc}$. The ratios $l_{0} / D_{0}$ for these cases correspond to 6.3 and 1 respectively as opposed to $\sim 40$ for galactic discs.

Integrating (1) gives the number of interceptors up to redshift $z_{i}$ as

$$
\mu\left(z_{i}\right) \equiv \int_{0}^{z_{i}} \frac{d \mu}{d z} d z=\left(\frac{D_{0}}{l_{0}}\right) \frac{\left[q_{0} z_{i}-\left(1-3 q_{0}\right)\right]\left(1+2 q_{0} z_{i}\right)^{1 / 2}+\left(1-3 q_{0}\right)}{3 q_{0}^{2}}
$$

We expect that on a random distribution of intervening galaxies, the absoption systems in the given sample will show a multiplicity distribution as per the Poissonian law. That is, the expected number with multiplicity $n$ will be given by

$$
\left\langle f_{n}(N)\right\rangle=\sum_{i=1}^{N} \frac{\left[\mu\left(z_{i}\right)\right]^{n}}{n !} \exp \left\{-\mu\left(z_{i}\right)\right\}, \quad n \geq 0
$$

Where the comparison is made with samples of QSOs which already contain at least one absorption system we modify (6) to

$$
\left\langle f_{n}\left(N^{\prime}\right)\right\rangle=\sum_{i=1}^{N^{\prime}} \frac{\left[\mu\left(z_{i}\right)\right]^{n}}{n !}\left[\exp \left\{\mu\left(z_{i}\right)\right\}-1\right]^{-1}, \quad n \geq 1
$$


Here $N^{\prime}$ is the number of QSOs in the sample $\left(N^{\prime}<N\right)$ with at least one absorption system.

It is likely that the nature of absorption systems places limits on the redshift ranges of the absorbers. We will consider such situations explicitly in this paper. In the case of a lower limit $z_{\min }$, say, the integral in (5) is zero for $z<z_{\min }$ and is an upper limit on the real value of $\mu(z)$ if $z>z_{\min }$. However, as we shall see, the adoption of the higher value given in (5) tilts the test in favour of the IGH.

We will compare the expected distribution (7) with the observed one at face value. We will then consider possible modifications of ( 7$)$ due to selection effects etc. that might affect such a comparison. We will also consider models with number density evolution of the absorbers, of the kind $n(z) \propto(1+z)^{\gamma}, \gamma=$ constant. Such models have been invoked, for example, by Sargent, Boksenberg and Steidel ${ }^{9}$, and by Sargent, Steidel and Boksenberg ${ }^{8}$.

\subsection{Comparison with the data}

We now compare the results derived above with the data as given in JHB'91. Following Burbidge et al ${ }^{5}$ we will first try three values of $R^{*}, R^{*}=20 \mathrm{kpc}, 40 \mathrm{kpc}$ and $100 \mathrm{kpc}$. We calculate the expected values of $\left\langle f_{n}\left(N^{\prime}\right)\right\rangle$ up to $n=10$ for these absorber sizes. We find that for $R^{*}=20 \mathrm{kpc}$ and $40 \mathrm{kpc}$, the two distributions match well within statistical fluctuations. For $R^{*}=100 \mathrm{kpc}$, however, the distribution generates a long tail of $n \geq 10$ not found in the sample. This suggests that the value $R^{*}=100 \mathrm{kpc}$ may be already be too large in realistic terms. Nevertheless, in what follows we will consider all three values of $R^{*}$ while comparing (7) for $N^{\prime}=315$ with 
the observed numbers.

Figure 1, shows the histogram of observed multiplicities along with those of the theoretical distribution (7) for $R^{*}=20 \mathrm{kpc}, 40 \mathrm{kpc}$ and $100 \mathrm{kpc}$. Broadly speaking the theory predicts relatively fewer multiple systems $(n>1)$ compared to single systems $(n=1)$, although the trend towards more multiple systems naturally increases as the interception cross-section increases. This is clear from the changes in the histogram as $R^{*}$ increases.

What happens if we introduce evolution? By having $\gamma=1,2$ we have generated similar theoretical distributions for comparison with the sample. Again the same qualitative effect is seen, viz, the multiple systems are far more abundant than predicted except for $R^{*}=100 \mathrm{kpc}$. Precise statistical analyses confirm this visual impression.

Table 1 gives the results at a glance through the computation of the probabilities for the Kolmogorov - Smirnov statistics. Notice that whether or not there is evolution, the difference between theory and observation is highly significant. We find that while the gap between our theoretical model and the observed data narrows as $R^{*}$ increases, the probability of the latter arising from a random fluctuations of the former is extremely small. In the case of $R^{*}=40 \mathrm{kpc}$, it may be possible to reduce the discrepancy by taking an evolutionary index $\gamma \sim$ 1 but for $R^{*}=100 \mathrm{kpc}$ evolution seems to make the discrepancy greater.

We will now explore the reasons for the observed discrepancy and discuss whether they affect the validity of the IGH. The reasons could be either due to a violation of the assumption of the random distribution of intervening galaxies that 
vitiates the assumption of a poisson distribution (6) or (7), or to subtle selection effects that may have crept into an incomplete and inhomogenous sample.

\subsection{Effect of Clustering}

The randomness assumption fails if the absorbers are significantly clustered, e.g., in groups or clusters of galaxies in which case we would expect to find more multiple systems compared to the poissonian prediction. In particular, if the clustering is characteristically over a size $r_{0}$ then we expect the lines to be clustered over redshifts corresponding to the distance $r_{0}$. The clustering phenomenon is generally described in terms of the two point correlation function, $\xi(r)$, being the excess probability of finding a typical galaxy separated from the given galaxy by a distance $r$ over the poissonian expectation value in a given volume. An emperical relation for $\xi(r)$ was given by Totsuji and Kihara ${ }^{18}$ and Peebles ${ }^{19}$. The function $\xi$ is proportional to $\left(r / r_{0}\right)^{-1.8}$ and is unity for $r \geq r_{0} \sim 5 h_{0}^{-1} M p c$ (Peebles ${ }^{19}$, Davis $\&$ Peebles $^{20}$, De Lapparent et $\mathrm{al}^{21}$ ).

A way of testing this effect is through Figure 2a, b with plots of histograms of separations between absorbers for each QSO having multiple systems, assuming that these absorbers are located at their redshift distances. Fig. 2a shows the entire distribution for 206 QSOs with multiple systems : it extends well beyond $1000 \mathrm{Mpc}$. Fig. 2b, however, is the subset of Fig. 2a confined to distances upto $\sim 300 \mathrm{Mpc}$ 
within which most of the data $(78.5 \%)$ reside. It is clear that the tail of this distribution far exceeds the characteristic cluster size or the typical length scale $\left(r_{0} \sim 5 \mathrm{Mpc}\right)$ of the two point correlation function for galaxies. For example, of all the pairs in Fig. $2 \mathrm{~b}$, as many as $87.8 \%$ lie beyond $10 \mathrm{Mpc}$.

It is noteworthy to mention here that, $\mathrm{Crotts}^{22}$, calculated the two point correlation function for metal-line absorption redshifts and found that the spatial $\xi(r)$ of QSO metal systems is flat beyond $r=2 h_{0}^{-1} M p c$ with a spike at $r<$ $2 h_{0}^{-1} M p c$. Nevertheless, to eliminate any possible effect of clustering of galaxies, all multiple absorption systems in a typical quasar occuring due to absorbers within a distance of $20 \mathrm{Mpc}$ of one another were considered as one and the multiplicities were reduced accordingly. The Kolmogorov-Smirnov probabilities for analyses șimilar to those of $\S 2.2$ for the 20,40 and $100 \mathrm{kpc}$ absorber sizes continue to be very low, being $7.92 \times 10^{-41}, 5.81 \times 10^{-11}$ and $5.49 \times 10^{-55}$ respectively. It is therefore evident that clustering on scales upto $20 \mathrm{Mpc}$ is not responsible for the observed excess multiplicities compared to what is expected from a Poisson distribution.

\subsection{Selection Effects}

It is clear that there is a significant discrepancy between the predictions of the IGH and the observed frequency of multiple systems. This had been the prima-facie finding of Burbidge et $\mathrm{al}^{5}$ and also later by Khare-Joshi and Perry ${ }^{23}$. We can turn the problem around and ask: What possible selection effects may be responsible for this discrepancy, assuming that the IGH is basically a sound and acceptable hypothesis? We now discuss the possibility that the inhomogenity of the databases 
conspires to work against the IGH. The following points suggest themselves.

(i) First we discuss the implication of the fact that we are not dealing with a 'complete sample'. For example, have all the QSOs in the JHB'91 been looked for absorption lines within specified wavelength ranges or with equal efficiency? The answer is 'no'. Also, the extent of time devoted by an observer (or several observers) to a quasar has depended on how interesting the quasar has been : those deemed particularly interesting or unusual have been looked at in more detail. Thus one has to allow for this subjective aspect. However, we can ask : What should we expect to see if all QSOs were looked at in detail?

The quasars in the JHB'91 sample show $f_{n} / f_{1}$ far higher for $n>1$ than expected in the case of $R^{*}=20 \mathrm{kpc}$ and $40 \mathrm{kpc}$. Since all entries in the JHB'91 catalogue are confirmed cases of absorption this implies that a march towards completion or a more detailed observation of each quasar can only produce higher values of $f_{n}$ for $n>1$ and so will increase the discrepancy between the theoretical and the observed values. However, for high values of $R^{*}$, e.g., $R^{*}=100 \mathrm{kpc}$ one can still argue that with detailed studies of quasar spectra the discrepancy between the observed and IGH-predicted ratios $f_{n} / f_{1}$ may disappear.

The sizes $R^{*}=20-40 \mathrm{kpc}$ are reasonable for galactic discs and halos. The value $R^{*}=100 \mathrm{kpc}$ is already excessive if one wants to argue that normal heavy elements are present out to such distances. The so called dark matter argument from flat rotation curves requires us to postulate nonbaryonic matter (as opposed to the normal one) at such distances, given the limits imposed by the big bang nucleosynthesis arguments. Thus theoretical justification will be required for the 
presence of metals at distances far exceeding the luminous radii of galaxies. It is worth noting that such a justification is more readily available in the quasi-steady state cosmology of Hoyle et al ${ }^{24,25}$ which allows for the dark matter to be baryonic and made of burnt out very old stars.

We next refer back to our comment in the introductory section on $\mu(z)$ being overestimated by (6). If we use the more realistic estimates dictated by the narrower redshift windows the values predicted by theory would come down, thus increasing the observed discrepancy for $R^{*}=20-40 \mathrm{kpc}$.

(ii) Perhaps a more positive way to approach the IGH is to consider ratios of the theoretically predicted values of $f_{n}$ [based on formula ( $(\bar{i})$ ] to those observed, in the JHB' 91 catalouges, and then to vary the absorber size to obtain the best fit. Figure 3 illustrates these ratios for absorber sizes ranging from $40 \mathrm{kpc}$ to 100 kpc in case of JHB'91. As found earlier, for $40 \mathrm{kpc}$ the observed frequencies at large multiplicities are systematically higher than theoretical ones. The situation improves with larger sizes upto $\sim 70 k p c-80 k p c$ and then worsens with further increase in size.

This visual impression is again borne out by statistical analyses. Thus the Kolmogorov - Smirnov test gives the largest probability of 0.02 for $R^{*}=70 \mathrm{kpc}$. The $\chi^{2}$ - test also favours this value with a normal $\chi^{2}$ probability of 0.0311 and reduced $\chi^{2}$ probability (Yate's correction) of 0.0507 . We have also considered the case of evolution of the type $(1+z)^{\gamma}$ with $\gamma=1,2$. In the case $\gamma=1$, the best fit value for the absorber size turns out to be $50 k p c$ with a Kolmogorov-Smirnov probability of 0.015 and $\chi^{2}$ probability of 0.07 (normal) and 0.11 (reduced). But, we 
could not get any good agreement for the range of absorber radii from $50-100 \mathrm{kpc}$ if the evolutionary index is considered to be 2 . From this analysis we infer that for the case of no evolution $(\gamma=0)$ in the absorber number density a tolerable agreement between the theory and observations can be obtained for the absorber radii of $70 k p c$ while for the $\gamma=1$ evolution case the optimum size comes down to $50 k p c$.

(iii) If the IGH is right, then with increasing $z_{e m}$ we should see more and more multiple systems. How does this prediction compare with the data? To test this expectation we perform the following analysis. For each QSO in the sample, i.e., for each given $z_{e m}$ and a specified $R^{*}$, calculate the probabilities of finding $1,2, \cdots, 10$ absorption redshifts through the IGH by using (7). These probabilities are normalised so that

$$
\sum_{i=1}^{10} p_{i}=1
$$

Define

$$
P_{i}(i \geq 1)=\sum_{j=1}^{i} p_{j}, \quad P_{0}=0 .
$$

Now, using a random number generator generate a random number between 0 and 1. If it lies between $P_{i-1}$ and $P_{i}(1 \leq i<10)$, then the QSO falls in the $i^{\text {th }}$ bin and is presumed to have absorption systems with multiplicity i. For the same QSO perform a large number of such trials (say 100) and take the mean value $\langle i\rangle$.

Figure 4 shows the distribution of multiplicities with respect to redshifts $z_{e m}$ 
from the JHB' 91 sample for $R^{*}=70 \mathrm{kpc}$. Note that as expected, the theory predicts a systematic increase in multiplicity with the emission redshift. The actual data, however, show no such trend. In fact, for $1.5 \leq z_{\mathrm{em}} \leq 3.5$ the observed multiplicity redshift distribution is a scatter diagram. Clearly, if the IGH is correct, a subtle but a systematic selection effect must be operating that would transform the theoretical trend into the observed scatter. Observers may be able to shed light on what this effect could be.

(iv) As an attempt to emperically estimate the selection effect that favours larger multiplicities we tried an extra ad-hoc factor $n^{\alpha}(\alpha>0)$. appearing in each term on the right hand side of (7). For a wide range of values of $\alpha \leq 4$ the theoretical biased distribution was compared with observations. There was, however, no significant improvement in the fit.

(v) We have already seen the effects of invoking evolution of the $(1+z)^{\gamma}$ type. Evolution does not help in producing a dramatic rise in the predicted multiplicity of redshift systems, even when tried along with (iv). In any case any emperical rule of evolution invoked by observers to bridge the gap between theory and observations, remains ad-hoc unless it can be ralated to the underlying physical scenario.

Nevertheless, to make our sample more homogeneous, we will next confine our attention to absorption lines of a specified rest wavelength. In particular . we will discuss the Mg II and C IV systems. For example, Young, Sargent and Boksenberg ${ }^{26}$ and Sargent, Steidel and Boksenberg ${ }^{8}$ and Sargent, Boksenberg and Steide $l^{9}$ have considered samples confined to only these absorption lines. Since the rest wavelength of the line is known and fixed we can consider a different approach 
to test the IGH.

\section{The Redshift Distribution of Mg II and C IV Absorption Systems}

\subsection{Theory}

Consider a shell of space centred on the observer and containing galaxies with redshifts in the range $(z, z+d z)$. Suppose each galaxy is spherical with radius $R$. Then the solid angle subtended by the galaxy at the observer is given by

$$
\Delta \Omega=\frac{\pi R^{2}(1+z)^{4}}{D(z)^{2}}
$$

where $D(z)$ is the luminosity distance corresponding to redshift $z$. Next suppose that there are $n$ such galaxies per unit volume. Assuming that they do not overlap, the total solid angle subtended by them at the observer is

$$
d \Omega=\frac{\pi R^{2}(1+z)^{4} n}{D(z)^{2}} V(z) d z
$$

where $V(z) d z$ is the volume of the shell. Using the well known formulae (cf. Narlikar ${ }^{27}$ for example) we get

$$
\frac{d \Omega}{4 \pi}=\pi R^{2}\left(\frac{c}{H_{0}}\right) \frac{n d z}{(1+z)^{2} \sqrt{1+2 q_{0} z}}
$$

Now $d \Omega / 4 \pi$ is the chance of interception of light coming from a quasar of redshift $z_{i}>z$ towards us. Hence if we have a sample of $N$ quasars with redshifts $z_{1}, \ldots, z_{N}$ then we expect absorption lines to have a redshift distribution $B(z) d z$, where 


$$
B(z)=\pi R^{2}\left(\frac{c}{H_{0}}\right) \sum_{z_{i}>z} \frac{n(z)}{(1+z)^{2} \sqrt{1+2 q_{0} z}}
$$

Taking typical sizes as $R \sim 50 \mathrm{kpc}=0.05 \mathrm{Mpc}, n_{0} \equiv n(z=0) \approx 0.1(\mathrm{Mpc})^{-3}$, we get

$$
B(z)=2.35\left(\frac{R}{50 \mathrm{kpc}}\right)^{2} h_{0}^{-1} \sum_{z_{i}>z} \frac{\left[n_{0} / 0.1 \mathrm{Mpc}^{-3}\right](1+z)}{\sqrt{1+2 q_{0} z}} .
$$

where $H_{0}=100 h_{0} \mathrm{~km} \mathrm{~s}^{-1} \mathrm{Mpc}^{-1}$.

This theoretical distribution of galaxy interceptions can be translated into the redshift distributions of given absorption lines. For example, if we consider the C IV $(\lambda=1548 \AA)$ line we should expect the above distribution of these absorption line redshifts to apply over a redshift range 1.26 to 3.5 for the optical window $3500-7000$ $\AA$, the upper bound being governed by the atmospheric bands that start coming into the spectra. Likewise for the $\mathrm{Mg}$ II $(\lambda=2800 \AA)$ line the relevant redshift range is $0.25-1.5$. We will see how $B(z) \delta z$ given by (14) compares with the observed histogram of redshifts for $\delta z=0.01$ (say).

\subsection{The Data}

We will consider the JHB'91 catalogue with special reference to the C IV and $\mathrm{Mg}$ II lines only. For theoretical computation we take $g_{0}=1 / 2$.

(i) Mg II line systems : Figure 5 shows the predicted and observed distributions one below the other. In both cases we have looked at the truncated redshift range 0.25-1.5 mentioned above. In Fig.5b we have the observations in histograms with 
redshift bins $\delta z=0.01$, the theoretical formula (14) having been multiplied by $\delta z$ and plotted in Fig.5a for comparison.

The shape of the histogram of observed redshifts of Figure $5 \mathrm{~b}$ looks similar to the continuous distribution predicted by (14) in Figure 5a. But the total expected number of absorbers is $\sim 664$ whereas the observed number is 238. This discreapnicy can be explained by the arguement of the incompleteness of the sample and it can be argued that with better resolution this gap can be bridged. However, if we assume that the incompleteness of the sample has systematically produced a deficient sample at all redshifts then uniformly scaling the theoretical distribution to the observed one gives a Kolmogorov-Smirnov probability of 0.15 . Thus by so correcting for incompleteness in the case of $\mathrm{Mg}$ II absorbers we find a modest agreement between the IGH and the observed data.

But, we do not need to resort to the incompleteness arguement if we bring the expected number down by making the sizes of the absorbers smaller with $R \sim 30 \mathrm{kpc}$. This resolution of the discrepancy, however, seems to be in contradiction with the results obtained from the multiplicity distribution of absorbers in the first part of the paper. The multiplicity argument required the absorbers to be $\geq 50 \mathrm{kpc}$.

A comment is needed on the shape of Figure 5 at the of low redshift end where we observe a deficit of absorption lines. Even allowing for the fact that we expect absorption redshifts of $\mathrm{MgII}$ systems only at $z \geq 0.25$, in Fig.5b we find far fewer systems at this end than expected in Fig.5a. Why are so many cases missed out? A possible explanation could be that approaching the redshift $\sim 0.25$, the QSO spectra have much poorer signal to noise ratio than at longer wavelengths. This will lead 
to fewer detections of absorption systems of $\mathrm{MgII}$ at redshifts $\sim 0.25$. This effect therefore could possibly account for the differences in the shapes of the two curves in Fig. 5a,b. There could be other reasons also. For example, we may be living in a local hole extending to, say, $z \approx 0.5$. Or we may need to invoke some evolution that makes $n$ very small for $z \leq 0.5$ and large for $z \geq 1$.

(ii) CIV line systems : Figure 6 shows the line distributions predicted (Figure 6a) and observed (Figure 6b). Again the redshift range is limited to $1.26-3.5$ for reasons mentioned at the end of $\S 3.1$. In this case the expected distribution neither matches in shape nor in total number. For example, the total number predicted is 920 as against 517 observed. Moreover, like in Mg II case there is a paucity of absorbers at low redshifts than expected. There is, however, an additional comment that nay be applicanto t:-

The redshift range $1.26 \lesssim z \lesssim 3.52$ for finding C IV lines overlaps the redshift range $z \gtrsim 2$ at which the Ly $\alpha$ systems begin to come in. This may lead to problems of indentification of C IV lines in specific cases. To avoid these cases we have shown in Figure 7 the theory vs. data for only those QSOs whose emission redshifts do not exceed 2. There are 44 such QSOs in the Junkkarinen catalogue. In this case, the expected number of absorbers (92) falls short of the actual oberved numbers (108) . This discrepancy can't be resolved by invoking a redshift dependant incompleteness since we already have more detections than predicted. We can, however, salvage the situation for the IGH by increasing the value of $R$ (Note that in the case of $\mathrm{MgII}$ as well as C IV in the previous case, we need to lower the value of $R$. Moreover an attempt towards completeness in the $\mathrm{Mg}$ II case will further worsen 
the case for IGH). Comparison between the normalised theoretical distribution and observed redshift distribution of absorbers gives a KS probability of $1.41 \times 10^{-2}$, which though better than in the case where the full redshift range of CIV absorbers was considered, is too small to lend definite support to the IGH.

(iii) Evolution : Can evolution help us? It is hard to take account of this effect without knowing the physical processes involved. Despite its popularity with interpretors of extragalactic observations power law evolution hardly justifies this lack of knowledge. Following, however, the work of Sargent, Steidel \& Boksenberg ${ }^{8}$ and Sargent, Boksenberg \& Steidel ${ }^{9}$ we have considered $n(z) \propto n_{0}(1+z)^{\gamma}$ type evolution in the number density of absorbers as was done in $\S 2$. The parameters $R, h_{0}, n_{0}$ combine together to give a single unknown coefficient in (14). For a given $\gamma$ and for $q_{0}=0.5$ (say) choose this coefficient such that the area under the theoretical and observed curves are the same. Thus we write

$$
R^{2} h_{0}^{-1} n_{0}=\alpha(50 \mathrm{kpc})^{2} \times 1 \times 0.1
$$

and choose $\alpha$ in the above fashion.

Table 2 lists the values of expected and observed number of absorbers, $\gamma$ and the probability when the theoretical prediction is compared with the observations. The cases of no evolution $\gamma=0$, 'positive' evolution $\gamma=1.45$, and 'negative' evolution $\gamma=-1.2$ suggested by the above authors are considered. It is clear that negative evolution recommended for $\mathrm{C} \mathrm{IV}$ and positive $\gamma$ for $\mathrm{Mg}$ II does not improve the fit and except in the case of Mg II absorbers none of the other cases shown in Table 
3 would pass the statistical criteria for goodness of fit! However, positive evolution for C IV systems greatly improves the fit and the statistical agreement is acceptably good.

\section{Conclusion}

We have thus found a mixed success for the IGH vis-a-vis the largest compilation of absorption line data on the QSOs. Prima facie examination shows that absorption by galactic discs of radius $20-40 \mathrm{kpc}$ either in clusters or distributed completely randomly does not generate satisfactory agreement with the data on multiplicity of the absorption systems, even allowing for the incompleteness and inhomogenity of the JHB'91 sample. Larger absorbers of radii $\sim 70 \mathrm{kpc}$ without evolution (or $\sim 50 \mathrm{kpc}$ with $\gamma=1$ evolution) can, however, produce passable agreement. The question, however, is, whether the standard stellar nucleosynthesis and chemical evolution of galaxies can deliver metals (like $\mathrm{Mg}, \mathrm{C}$ ) as far out and beyond the visible boundary of a typical galaxy.

The IGH fares somewhat better when the redshift distributions of specific line systems in particular, C IV and Mg II are compared with the data. Again, prima facie, the agreement is not satisfactory, but it can be made so with due allowance for incompleteness of the sample, and by invoking evolution. The evolution invoked is ad hoc in the sense that it has not been linked with the astrophysical evolution of the universe.

Thus while there is no conclusive evidence on the basis of this analysis in favour of the IGH the flexibility of theoretical parameters and the incompleteness 
of samples used allow sufficient margin of uncertainty for the hypothesis to survive. We therefore feel that other options like intrinsic absorption might also be explored along with the IGH, although we have not done so here.

Even though the present investigation has not proved decisive either way, the statistical techniques used here can be useful in returning to the issue in future as and when more controlled and larger samples become available, wherein the selection effects discussed and estimated here are adequately sorted out. It is because the IGH can provide a useful diagonstic on the state of the universe out to redshifts of $\sim 4$, that we strongly feel that statistical examination of this kind would be worth repeating on such future samples. Till then the issue of the origin of the metal absorption systems remains inconclusive with the IGH providing part but not the whole of the explanation .

Acknowledgement : We thank Vesa Junkkarinen, Adelaide Hewitt and Geoffrey Burbidge for use of their catalogue. DD was financially supported by a Senior Research Fellowship of Council of Scientific and Industrial Research. 


\section{References}

1. Bahcall, J.N. and Salpeter, E.E., 1965, ApJ, 142, 1677

2. Sargent, W.L.W. 1987, Observational Cosmology, ed. A. Hewitt et al. (Kluwer : IAU), 777

3. Wagoner, R.V. 1967, ApJ, 149, 465

4. Bahcall, J.N. and Spitzer, L. Jr. 1969, ApJ, 156, 463

5. Burbidge, G., O'Dell, S.L., Roberts, D.H. and Smith, H.E. 1977. ApJ, 218, 33

6. Roeder, R.C., 1969, ApJ, 157, L153

7. Sargent, W.L.W., Young, P.J., Boksenberg, A., Tytler, D. 1980, ApJS, 42, 41

8. Sargent, W.L.W., Steidel, C.C. and Boksenberg, A. 1988, ApJ, 334, 22

9. Sargent, W.L.W., Boksenberg, A. and Steidel, C.C. 1988, ApJS, 68, 539

10. Bergeron, J. and Boissè, P. 1991, A\&A, 243,344

11. Steidel, C.C. and Dickenson A. 1992, ApJ, 394, 81

12. Borgeest, U. and Mehlert, D. 1993, A\&A, 275, L21

13. Junkkarinen, V., Hewitt, A. and Burbidge, G. 1991, ApJS, 77, 203

14. Bahcall, J.N., AJ, 1971, 76, 283

15. Bahcall, J.N and Peebles, P.J.E. 1969, ApJ, 156, L7

16. Schechter, P. 1976, ApJ, 203, 297

18. Totsuji, H. and Kihara, T. 1969, Publ. Astron.Soc.(Japan), 21, 221 
19. Peebles, P. J. E. 1980, The Large Scale Structure of the Universe, (Princeton University Press : Princeton)

20. Davis, M. and Peebles, P. J. E. 1983, ApJ, 267, 465

21. De Lapparent, V., Geller, M. J. and Huchra, J. P., 1988, ApJ, 332, 44

22. Crotts, A. P. S. 1985, ApJ, 298, 732

23. Khare-Joshi, P. and Perry, J.J. 1982, MNRAS, 199, 785

24. Hoyle, F., Burbidge, G. and Narlikar, J. V., 1993, ApJ, 410, 437

25. Hoyle, F., Burbidge, G. and Narlikar, J. V., 1994, MNRAS, 267, 1007

26. Young, P., Sargent, W.L.W. and Boksenberg, A. 1982, ApJS, 48, 455

27. Narlikar, J.V. 1993, Introduction to Cosmology, $2^{\text {nd }}$ Ed. (Cambridge University Press : Cambridge) 


\section{Figure Captions}

Fig. 1 : Comparison of theoretical multiplicity distribution (based on no evolution) with the observed distribution of 315 QSOs showing absorption features in their spectra from the JHB'91. Three sizes of intervening absorbers are considered.

Fig. 2a : Histogram of the separations between 848 line pairs upto $2000 \mathrm{Mpc}$.

Fig. 2b : Enlarged view of the above histogram showing separations upto $300 \mathrm{Mpc}$.

Fig. 3 : Ratios of theoretically predicted $f_{n}$ to those observed, for absorber sizes ranging from $40 \mathrm{kpc}$ to $100 \mathrm{kpc}$.

Fig. 4 : Comparison of observed multiplicity - redshift $\left(z_{e m}\right)$ distribution with the theoretical one considering an absorber size of $70 \mathrm{kpc}$.

Fig. 5a: Theoretical distribution of Mg II absorbers for 178 QSOs in the JHB'91 plotted against redshit $z$. Lhe munber of galaxies per unit volume $(n)$ is taken to be constant $\left(n=0.1 \mathrm{Mpc}^{-3}\right)$. The absorption redshifts in the above plot do not include those with $\left|z_{e m}-z_{a b s}\right|<0.01$, which are commonly assumed to be intrinsic to the QSO.

Fig. 5b : Histogram showing the observed distribution of $272 \mathrm{Mg}$ II absorption systems found in the spectra of 178 QSOs in the JHB'91

Fig. 6a : Theoretical distribution of C IV absorption systems calculated from emission redshifts of 192 QSOs from the JHB'91 showing C IV absorption in their spectra.

Fig. 6b : Histogram of 521 observed C IV absorption systems given in the JHBC'91.

Fig. 7a : Theoretical distribution of C IV absorption systems in the redshift 
range of 1.26-2.0. This criterion was chosen to eliminate possible confusion with absorption systems within the Ly $\alpha$ forest.

Fig. 7b : The observed distribution of $108 \mathrm{C} \mathrm{IV} \mathrm{absorption} \mathrm{systems} \mathrm{in} \mathrm{the} \mathrm{redshift}$ range of 1.26-2. The distribution falls far short of the expected number calculated, as shown in (a) above. 
Table 1

Kolmogorov-Smirnov Probabilities for Theoretical Models vs Observations

\begin{tabular}{cccc}
\hline \hline $\mathrm{R}^{*}$ & no evolution & $(1+z)$ & $(1+z)^{2}$ \\
\hline 20 & $1.14 \times 10^{-97}$ & $1.15 \times 10^{-71}$ & $5.07 \times 10^{-32}$ \\
40 & $6.25 \times 10^{-38}$ & $3.80 \times 10^{-5}$ & $1.49 \times 10^{-16}$ \\
100 & $1.01 \times 10^{-23}$ & & $2.09 \times 10^{-80}$ \\
\hline
\end{tabular}


Table 6D

Kolmogorov-Smirnov Probabilities of Observed vs Normalised Theoretical Distribution for CIV and MgII absorbers.

\begin{tabular}{cccccr}
\hline \hline Case & Sample & Theoretical & Observed & $\gamma$ & \multicolumn{1}{c}{$P_{K S}$} \\
\hline No & CIV & 919.943 & 517 & - & $2.14 \times 10^{-6}$ \\
\multirow{2}{*}{ Evolution } & CIV (Lyman lim.) & 92.14 & 108 & - & $1.41 \times 10^{-3}$ \\
& MgII & 664.30 & 238 & - & 0.15535 \\
\hline \multirow{2}{*}{ Evolution I } & CIV (Lyman lim.) & 30.11 & 517 & -1.2 & $3.44 \times 10^{-18}$ \\
& MgII & 1690.33 & 108 & -1.2 & $9.80 \times 10^{-4}$ \\
& CIV & 4616.32 & 517 & +1.45 & $1.84 \times 10^{-13}$ \\
\hline \multirow{2}{*}{ Evolution II } & CIV & 359.508 & 108 & +1.45 & 0.47011 \\
& CIV (Lyman lim.) & 3529 & +1.45 & 0.1706 \\
\hline
\end{tabular}




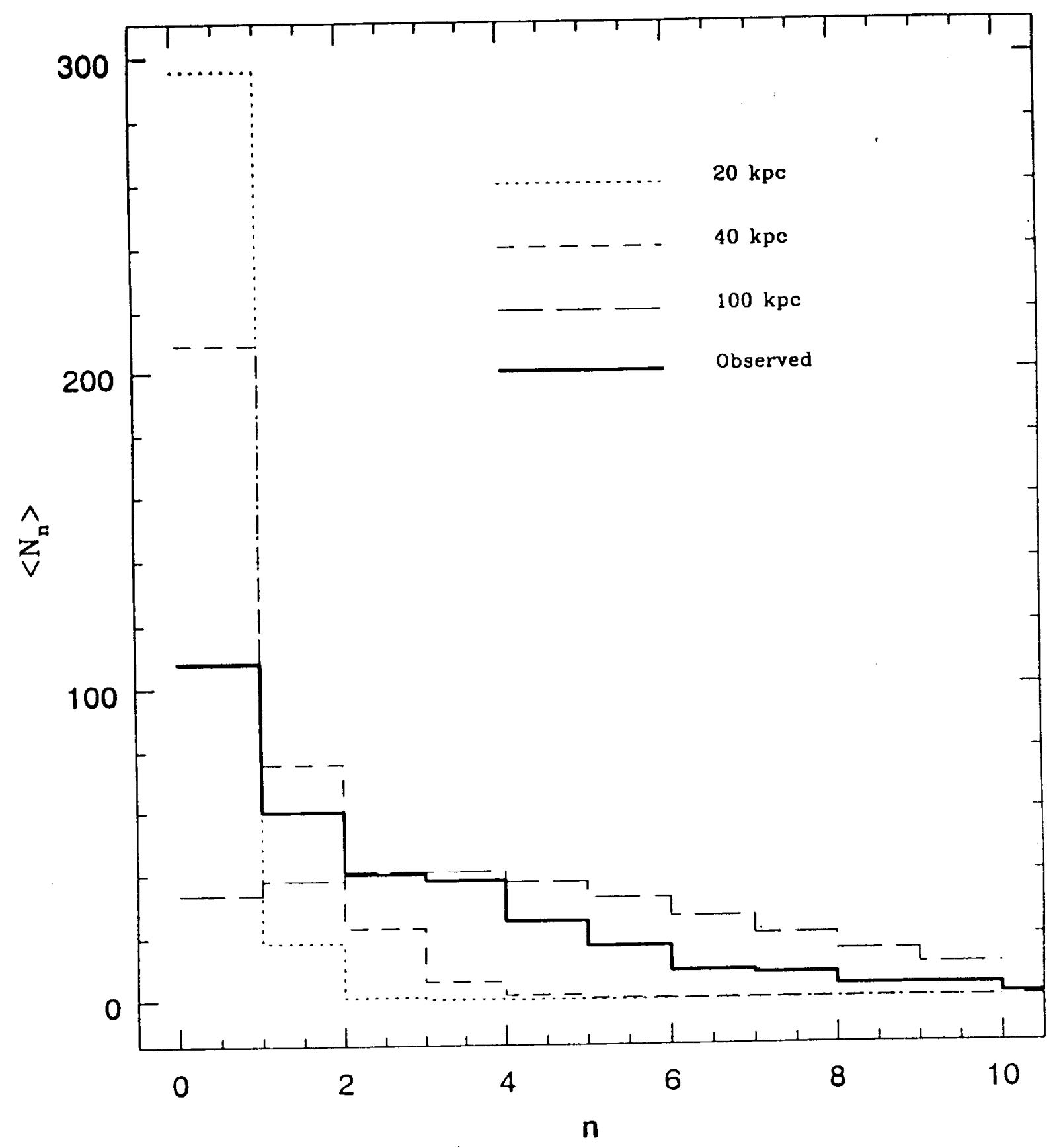

Figure 1.

The Intervening Galaxies Hypothesis of the Absorption Spectra of Quasi-Stellar Objects : some staistical studies.

Debiprosad Duari \& Jayant V Narlikar 

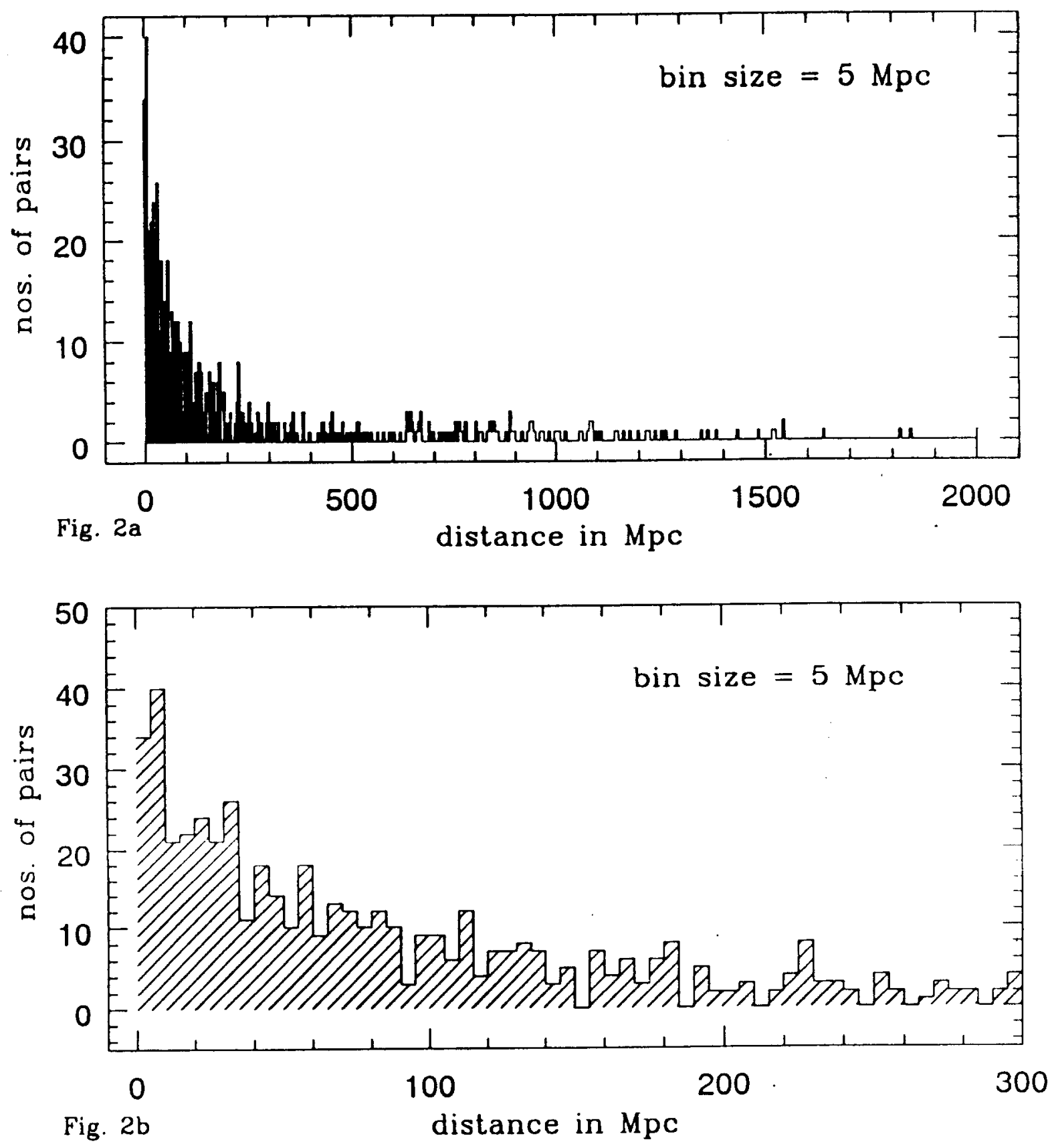

\section{Figure 2}

The Intervening Galaxies Hypothesis of the Absorption Spectra of Quasi-Stellar Objects : some staistical studies.

Debiprosad Duari \& Jayant $V$ Narlikar 


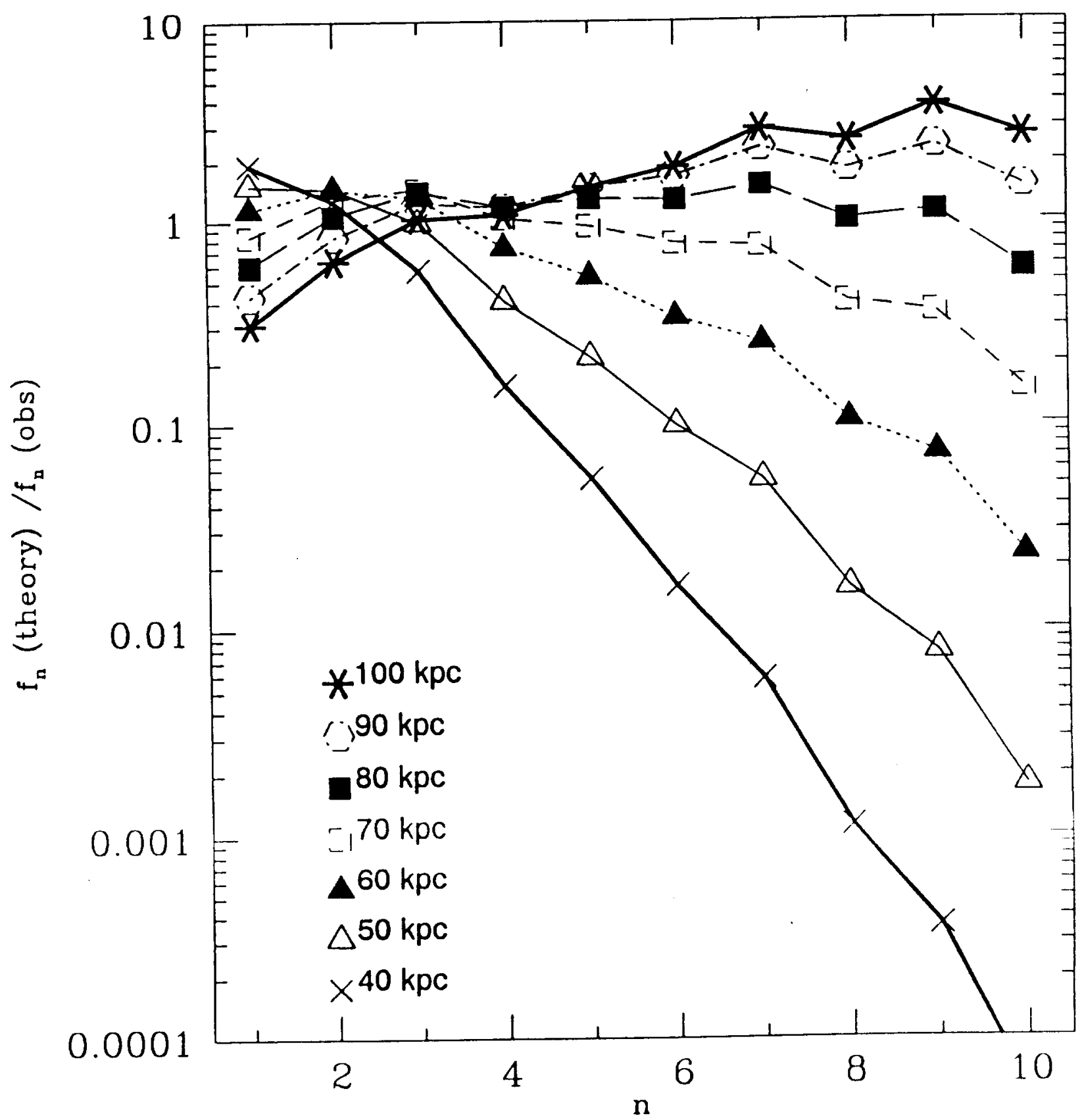

Figure 3

The Intervening Galaxies Hypothesis of the Absorption Spectra of Quasi-Stellar Objects : some staistical studies.

Debiprosad Duari \& Jayant V Narlikar 


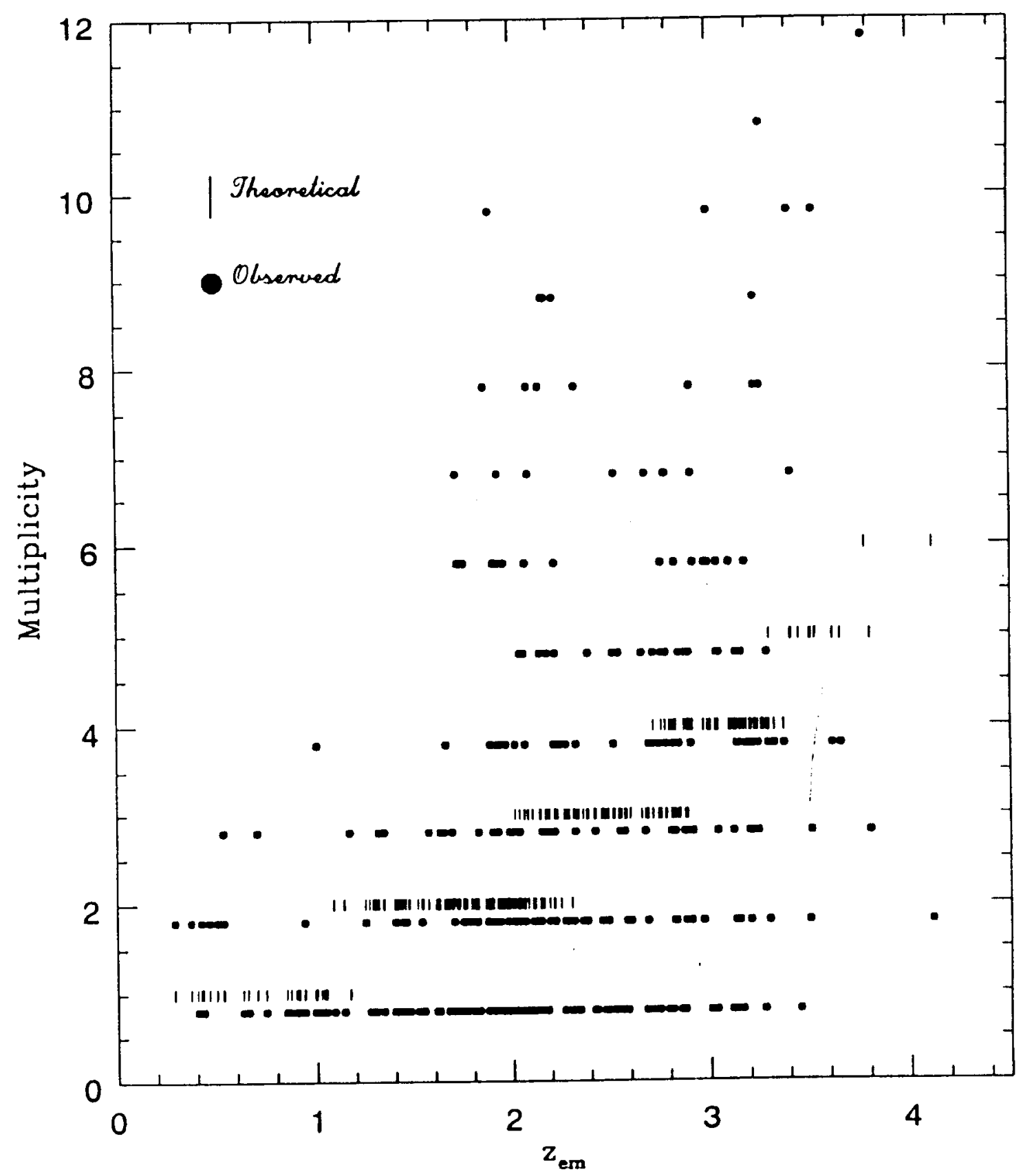

Figure 4

The Intervening Galaxies Hypothesis of the Absorption Spectra of Quasi-Stellar Objects : some staistical studies.

Debiprosad Duari \& Jayant $\mathbf{V}$ Narlikar 

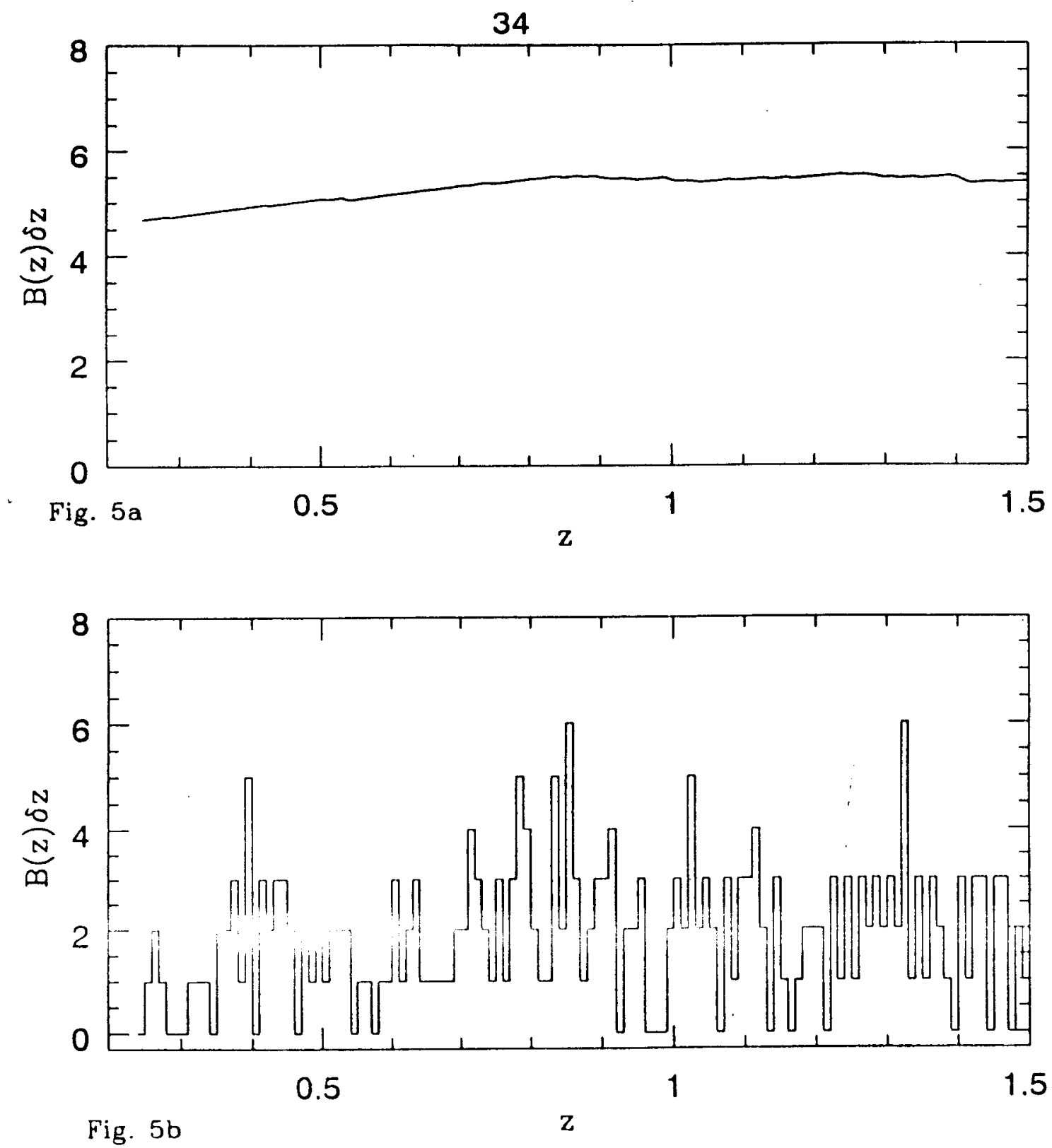

Figure 5

The Intervening Galaxies Hypothesis of the Absorption Spectra of Quasi-Stellar Objects : some staistical studies.

Debiprosad Duari \& Jayant V Narlikar 

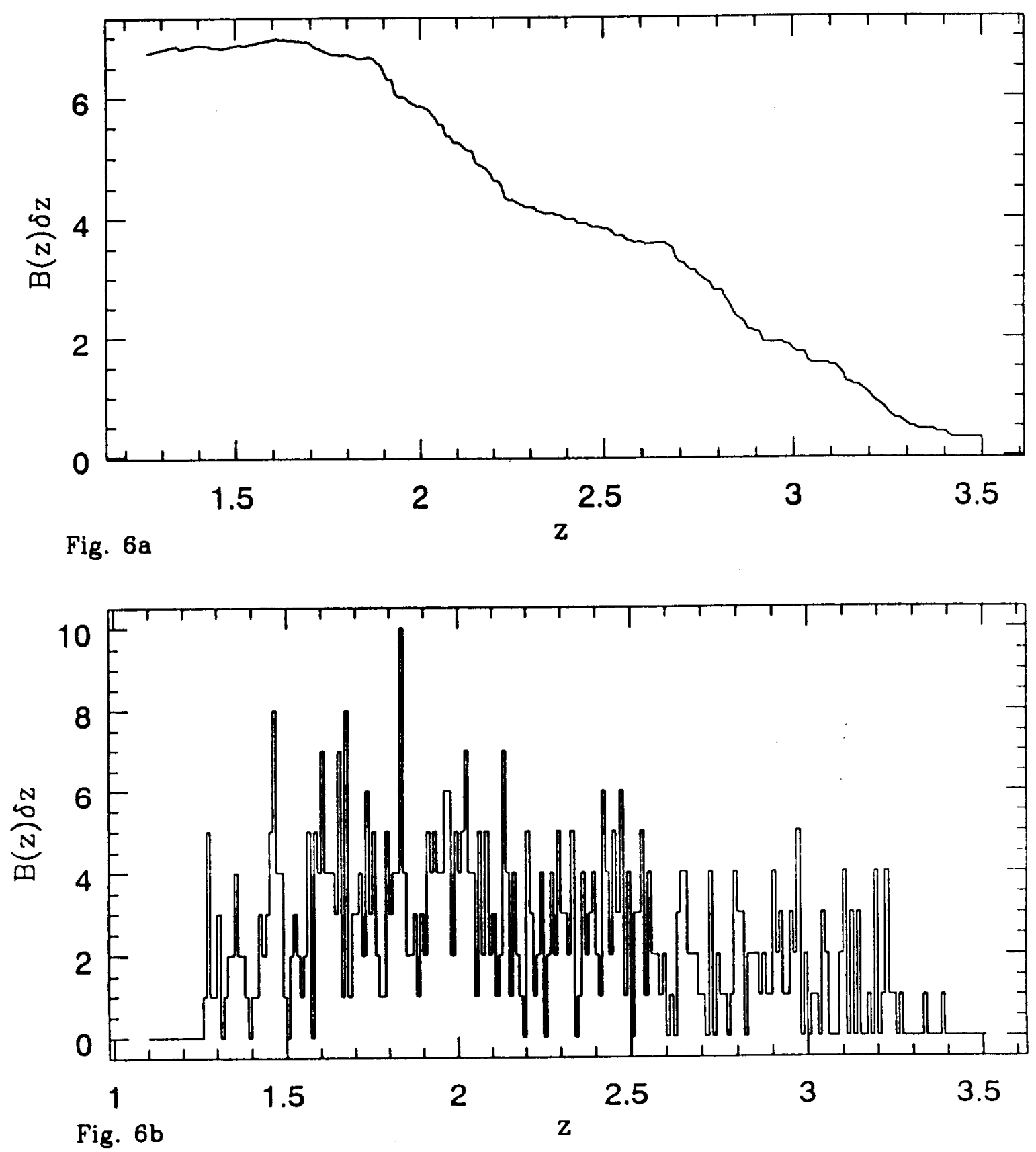

Figure 6

The Intervening Galaxies Hypothesis of the Absorption Spectra of Quasi-Stellar Objects : some staistical studies.

Debiprosad Duari \& Jayant V Narlikar 

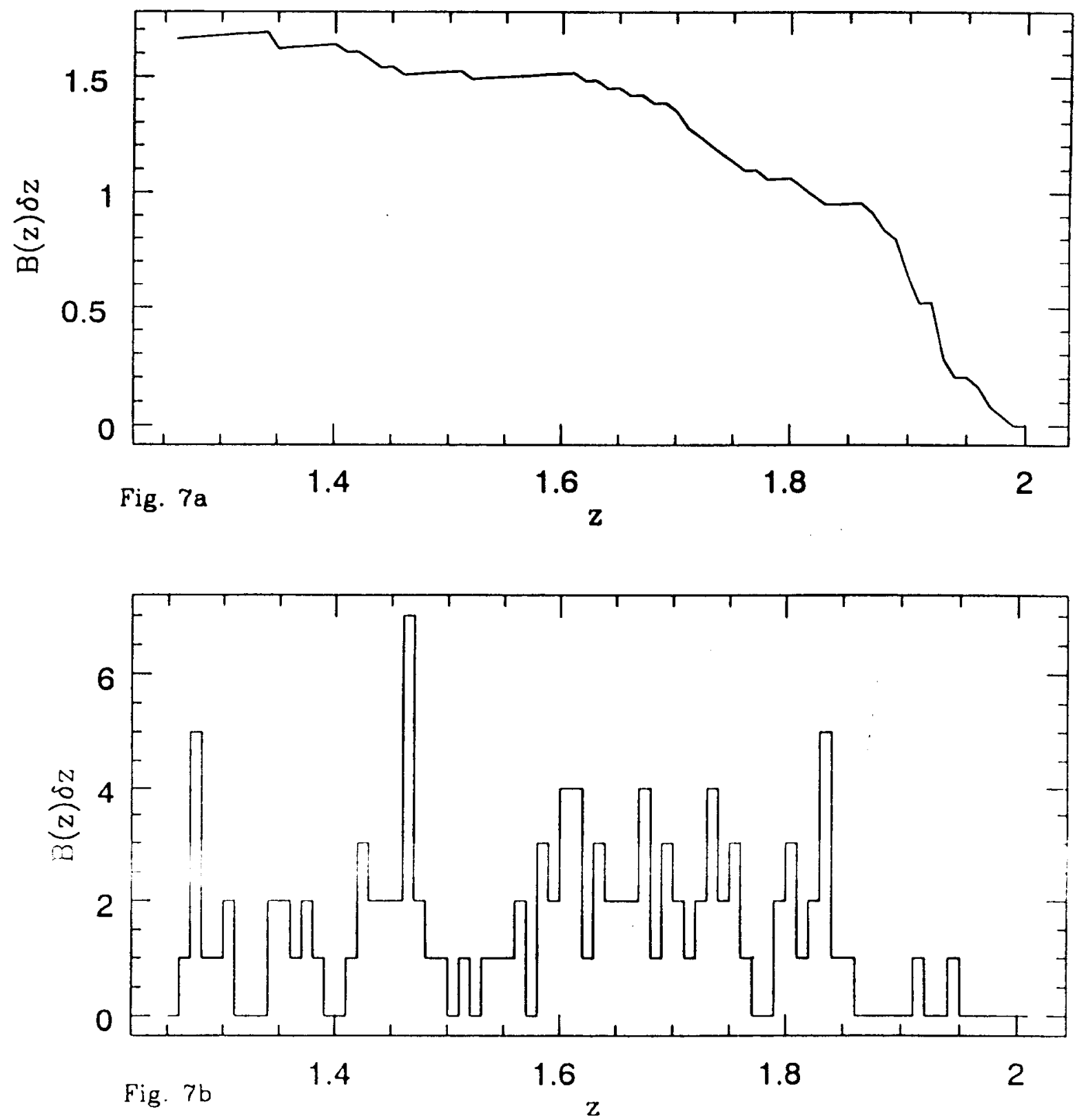

Figure 7

The Intervening Galaxies Hypothesis of the Absorption Spectra of Quasi-Stellar Objects : some staistical studies. Debiprosad Duari \& Jayant V Narlikar 